\title{
Development of community health service-oriented computer- assisted information system for diagnosis and treatment of respiratory diseases
}

\author{
Yuefang $\mathrm{Wu}^{1,2}$, Xin $\mathrm{Yao}^{1}$, Peili Sun ${ }^{1}$, Yong $\mathrm{Hu}^{2}$, Yuchuan $\mathrm{Zhu}^{2}$, Yin $\mathrm{Hu}^{3}$
}

\begin{abstract}
Objective: Community health services are an emerging trend. We have found in practice that diagnosis and treatment of respiratory diseases in the community are distinct. The respiratory department's daily work involves a number of outpatient registration items and a vast workload. The routine manual operation is inefficient and it is not convenient to make effective statistical analysis of the outpatient data to identify the risk factors closely related to diseases. Therefore, it is imperative to process the outpatient information of patients with respiratory diseases effectively and efficiently in a unified manner by means of computer technology.
\end{abstract}

Methods: The design and realization of the Community $\boldsymbol{H}$ ealth $\mathbf{S e r v i c e - o r i e n t e d ~ c o m p u t e r - ~}$ assisted Information System for Diagnosis and $\boldsymbol{T}$ reatment of $\boldsymbol{R}$ espiratory $\boldsymbol{D}$ iseases (CHS-DTRD) was completed as part of the community intervention study on bronchial asthma that was carried out jointly by the Nanjing First Hospital Affiliated to Nanjing Medical University and the Hospital of Nanjing University of Science \& Technology, and based on 2 years of experience and the needs of an overall analysis.

Results: The computer-assisted information system for diagnosis and treatment was developed using Java Server Page (JSP) technology and introducing the advanced Asynchronous JavaScript XML (AJAX) technique and MS-SQL Server was used in the background database. CHS-DTRD was composed of eight functional modules (outpatient data maintenance, outpatient appointment, intelligent analysis for disease risk factors, query and statistics, data dictionary maintenance, database manipulation, access control, and system configuration). CHS-DTRD featured a friendly interface, convenient operation, and stability and reliability.

Conclusion: Community health-oriented diagnosis and treatment of respiratory diseases is simple, programmable, and intuitive, thus the workload of physicians is significantly reduced and the work efficiency is improved. This system facilitates an intelligent analysis of disease risk factors using data mining technology, and provides physicians with suggestions on intelligent analysis for diagnosis of disease and conclusion of disease causes.

Keywords: Community health service, Respiratory diseases, Computer-assisted diagnosis and treatment, Intelligent analysis, Browser/server
1. Department of Respiratory Medicine, the First Affiliated Hospital with Nanjing Medical University, Nanjing, Jiangsu Province, 210029, China

2. Nanjing University of Science and Technology Hospital, Nanjing, Jiangsu Province, 210094, China

3. Jiangsu Shengze Hospital, Suzhou, Jiangsu Province, 215228, China

CORRESPONDING AUTHOR: Xin Yao

Department of Respiratory Medicine, the First Affiliated Hospital with Nanjing Medical University, Nanjing, Jiangsu Province, 210029, China

E-mail: njyudj@njust.edu.cn

Funding: National Natural Science Foundation of China [Grant No. 61373062]; The Fundamental Research Funds for the Central Universities [Grant No. 30920130111010]; Social Development Project of Wujiang City, [Grant No. WS201217].

Received 16 September 2013; Accepted 17 November 2013 


\section{Introduction}

Because of the deterioration in living environments and the development of unhealthy living habits, such as smoking and aging of the social crowd structure, respiratory diseases, especially chronic respiratory diseases, have become the major cause of death and disability worldwide in the most recent 30-40 years. Currently, nearly one-fourth of deaths are caused by respiratory diseases [1]. Bronchial asthma and chronic obstructive pulmonary disease (COPD) are the most common chronic respiratory diseases. As one of the most common chronic diseases, bronchial asthma has a mortality rate of $1 \%-5 \%$ in China. Although the absolute morbidity of COPD is not recorded in China, according to a partial survey, the COPD morbidity in respondents $>15$ years of age is about $3.17 \%$ and the 10 -year mortality rate reaches $50 \%$, ranking third amongst various causes of death in China [2]. As shown by data from the National Ministry of Health in recent years, the morbidity of chronic respiratory diseases has an increasing trend.

In contrast, with the development of the social economy and increasing health needs, community health services are being developed rapidly in first-tier cities, such as Beijing, Tianjin, and Shanghai [3, 4], and gradually entering smalland medium-sized cities in China. The development of community health services not only enhances health fairness and efficiency, but also effectively improves the health status of residents and controls the increase in medical expenses [4]. Community health services are distinctive, which requires the community hospitals to install innovative hardware and software based on the health service requirements.

In recent years, the First Affiliated Hospital with Nanjing Medical University and the Nanjing University of Science and Technology Hospital conducted studies on chronic respiratory diseases based on community characteristics. It was shown that the community diagnosis and treatment of respiratory diseases is distinct. The respiratory department's daily work involves a number of outpatient registration items and a vast workload. The routine manual operation was inefficient and it was inconvenient to perform effective statistical analyses on outpatient data to identify the risk factors closely related to diseases. Although the Hospital Information System (HIS) [5, 6] has been widely used as an effective information-assisted diagnosis and treatment method in many hospitals in China, the HIS is a large-sized general software system. A large number of facility resources are required to run such a system, and it is apparent that primary level and community hospitals cannot afford this system. In contrast, special requirements for the management, diagnosis, and treatment of specific diseases (e.g., chronic respiratory diseases) have not been taken into consideration to ensure the universality of the software during its design stage. Thus, the HIS has to be re-developed as to include specific disease types. Such a comprehensive diagnosis and treatment system is very complicated, so such secondary development requires software engineers who are familiar with the HIS frame, and medical specialists to smooth the work flow; this requires consumption of large human and financial resources and is unbearable by the primary level or community hospitals $[7,8]$.

According to the characteristics of community health services, we developed a $\boldsymbol{C}$ ommunity $\boldsymbol{H}$ ealth $\boldsymbol{S e r v i c e - o r i e n t e d ~}$ Computer-assisted Information System for the Diagnosis and Treatment of $\boldsymbol{R}$ espiratory $\boldsymbol{D}$ iseases (CHS-DTRD) on the basis of a comprehensive survey on the characteristics of the clinical diagnosis and treatment for respiratory diseases. At the end of 2010, the project team had completed the design and realization of the stand-alone version of the computer-assisted information system for the diagnosis and treatment of respiratory diseases [7], which was used in the clinical diagnosis, treatment, and scientific study of respiratory diseases by the First Affiliated Hospital with Nanjing Medical University and the Nanjing University of Science and Technology Hospital. This system provides basic functional modules required by clinical application, but there are still shortcomings. First, the standalone version does not have a network function. Therefore, data-related operations, such as merger, release, switch, and summary, have to be completed by means of another memory medium. Second, it is inconvenient because the system operation and maintenance should be installed and updated on each machine. Third, ACCESS, as used in the background database, cannot satisfy the needs of rapid growth of the volume of data. In comprehensive consideration of the above elements and the shortcomings detected during $>1$ year use of the system, the project team developed WEB-based CHS-DTRD using advanced AJAX and JSP techniques, which further 
improved the module functions of the original system. The system was essentially improved with respect to convenience, accessibility, expansibility, and flexibility.

Community health services are an emerging trend. The diagnosis and treatment of respiratory diseases in communities are distinct. The daily work in the respiratory department involves a number of outpatient registration items and a vast workload. The routine manual operation is inefficient and it is inconvenient to perform effective statistical analyses of the outpatient data to identify the risk factors closely related to diseases. Therefore, it is imperative to process the outpatient information of patients effectively and efficiently in a unified way by means of computer technology.

\section{Methods}

(1) Overall analysis of system requirements: The success of a system mainly depends on a understanding of the requirements. Therefore, an overall analysis on the requirements of CHS-DTRD was completed on the basis of the practice in the most recent 2 years regarding the background of the community invention study program of bronchial asthma, which was carried out by the First Affiliated Hospital with Nanjing Medical University and the Nanjing University of Science and Technology Hospital.

(2) Application of advanced technology to develop a stable and efficient system: The project team adopted advanced AJAX, JSP, and MS-SQL server techniques to ensure the stability and efficiency of the system. These techniques were used as follows:

First, to improve the operating efficiency of the system and enhance the user experience, the AJAX technique was used. AJAX is a standardized and normalized technique which can be widely supported. No plug-in component or virtual machine is required. As a future standard of Web development, AJAX would greatly improve the availability of Web application and the interaction experience of users, relieve the burden of the server, and play an important role in the development of a Web application. CHS-DTRD with the AJAX [9] technique took the three-tier frame of the B/S mode and asynchronous interaction, which greatly relieved the burden of the server, reduced the waiting time for users, improved the working efficiency of the system, and provided a good experience for users.
Second, to improve the cross-platform extension capacity of the system, the JSP technique was used. The JSP standard was dominated by Sun [10] and developed according to the opinions of various manufacturers in the fields of computer software and hardware, communications, and databases. JSP is a dynamic Web page technique based on Java. When receiving a request for visiting the JSP webpage, the Web server would first implement the related program segment and return the implementation results to the client end in the form of HTML. The program segment could operate databases, reset webpages, and send E-mail. All program operations were implemented at the server end. Only the results obtained were sent to the client end through network uploading. JSP had few requirements for client browsers and could be visited without a plug-in component, ActiveX, Java Applet, and even without a Frame browser. JSP adheres to Java's spirit "write once, run anywhere." JSP is a technique irrelevant to a hardware platform, operating system, or Web server. Because of the openness of JSP, many manufacturers have developed JSP development tools and a JSP engine under many platforms, providing a basis for realization of platform independence. To improve the cross-platform capacity of the system, we used the JSP technique to develop server applications.

Third, to improve the data processing capacity of the system, the senior relational database system was used. The ACCESS small database used in the stand-alone version of the system [7] could not meet the increasing needs of data processing. In November 2005, Microsoft Corporation issued SQL Server 2005, an upgrade version of the database software, SQL Server. SQL Server 2005 significantly enhanced enterprise support, business intelligence application, and management and development efficiency [11]. The integrated data management and analysis platform provided by SQL Server 2005 could help the organization manage information from key businesses more reliably and operate complicated business applications more effectively. An enterprise could obtain better business performance and insight by using the integrated report and data analysis tools. From SQL Server 2000 to SQL Server 2005, the database system not only had realized higher performance and processing capacity, but also brought many new characteristics; however, these new characteristics 
were consistent with the development orientation of a modern database, such asthe support for XML and implant of senior language support in the SQL language. In an enterprise environment, the database system attached more importance to safe, high availability, and integrated management tools. MS SQL Server 2005 was easy to deploy and maintain, and showed a high cost-performance ratio, so it was used as the background database system in this study.

\section{Results}

\section{Overall system architecture}

Fig. 1 shows the system architecture of the CHS-DTRD based on the AJAX technique. The system is divided into three tiers, as follows: the first tier is the presentation tier located at the Web browser end for the purpose of interaction with users, such as collecting data from users and sending the data to the AJAX engine and the server end, and representing the results to users; the second tier is the Web server (the core of the system), where the business logic of functional modules is realized; and he third tier is the background database, which is used for data storage and persistence of business objects.

\section{Realization of functional modules of the system}

The CHS-DTRD is composed of eight main functional modules (outpatient data maintenance, outpatient appointment, intelligent analysis for disease risk factors, query and statistics, data dictionary maintenance, database manipulation, access control, and system configuration), as shown in Fig. 2.

Fig. 3 shows the initial welcome screen of the system. The buttons of the eight functional modules are arranged in order from top-to-bottom on the left. Click a button to enter the page of the relevant functional module. In addition, when entering the system, some information related to the user will appear in the middle of the page as "News and Messages" to help the user obtain closely-related information timely.

Outpatient data maintenance: When clicking "Outpatient Data Maintenance" on the left side of this page, the page of outpatient data maintenance can be entered, as shown in Fig. 4. On this page, two sub-functions as "New Outpatient Data Input" and "Old Outpatient Data Modification" are provided to facilitate outpatient data input. In addition, the integrality of patient information is of critical importance for the subsequent diagnosis and treatment and the analysis for disease risk factors. Therefore, the following were considered for development of the functions of the outpatient data maintenance module:

(a) Input convenience: The outpatient data input was speeded up and the patient waiting time was reduced by using data dictionary and shortcut keys, and dynamically giving the most input prompts; for revisiting patients, the system will automatically provide the previous diagnosis and treatment information to facilitate understanding of the patient treatment history and improve the diagnosis and treatment pertinence. The physician may also make a customized query of the previous outpatient information.

(b) Data rigor: Data rigor is a principle by which the information of the completed case is not modified, especially the information regarding prescribed medications. Should such information need modification, authorization from the superior user must be obtained and a physician shall only amend the information recorded. The amendment should be completely recorded in the background

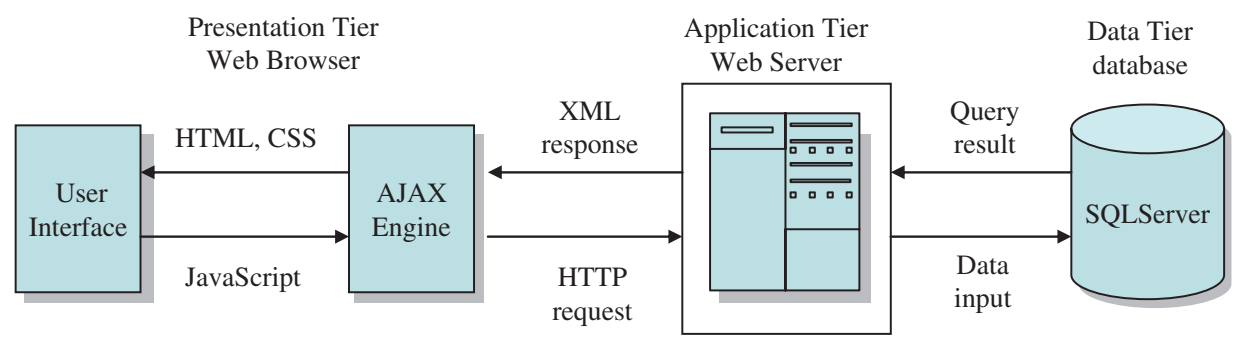

Fig. 1. System architecture of the community health service-oriented computer-assisted information system for the diagnosis and treatment of respiratory diseases based on the AJAX technique. 


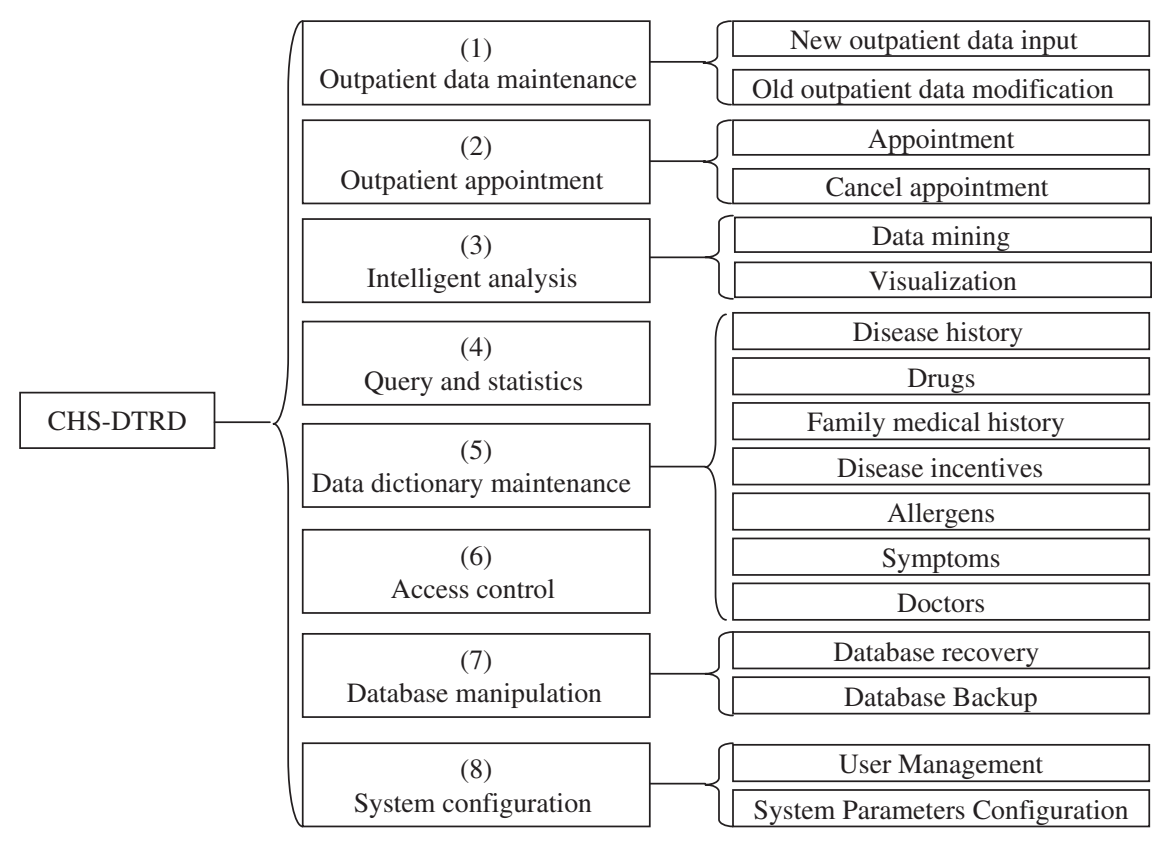

Fig. 2. Eight functional modules of the CHS-DTRD.

job log to provide true historical diagnosis and treatment information and prevent falsification.

(c) Flexible support for management of respiratory diseases: The useful forms and classified diagnosis and treatment guidelines required for chronic respiratory diseases are provided and may be upgraded with the disease diagnosis and treatment progress. During data input, relevant guidelines or formula may be clicked based on the symptoms and test results to give the patient diagnosis and disease stage and level, and assist the physicians and nurses in implementing standard diagnosis and treatment.

Outpatient appointment: This system also realizes a community health service-oriented workflow-based online outpatient appointment system to facilitate the outpatient appointment and improve the hospital work efficiency. All

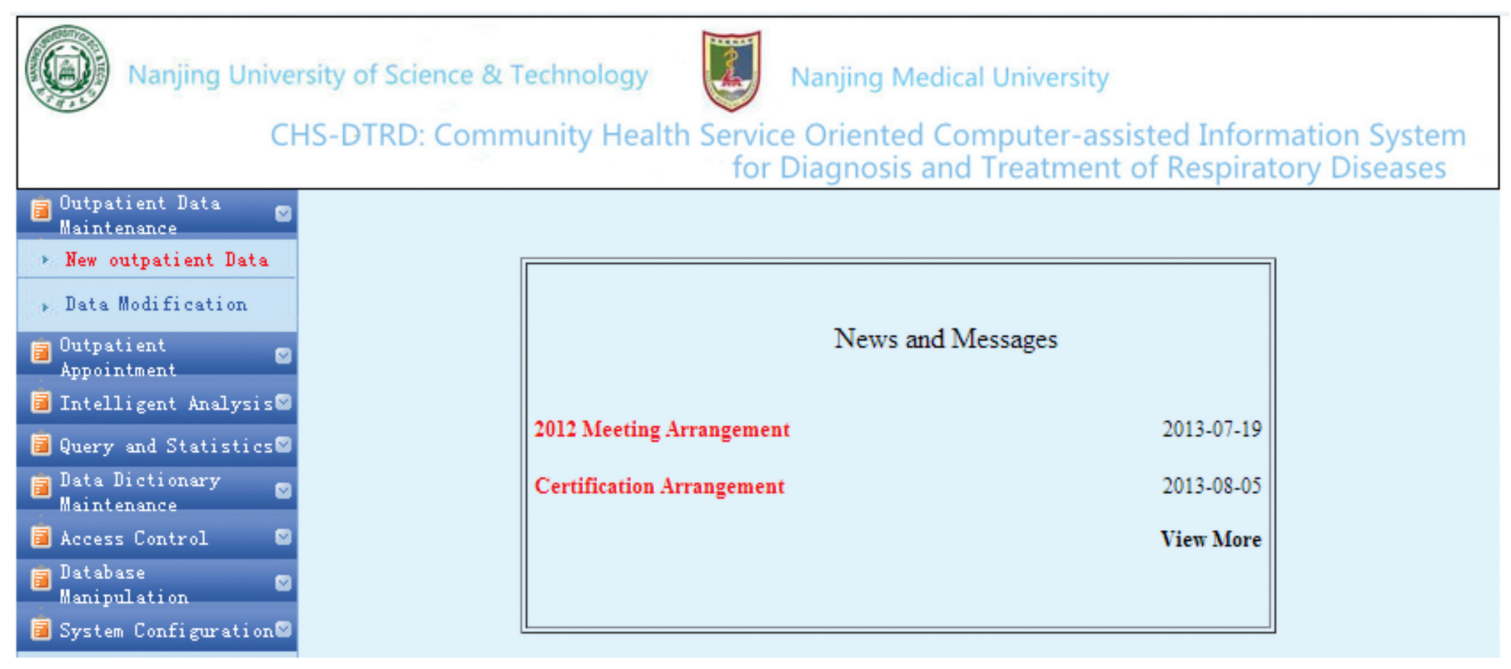

Fig. 3. The Welcome Page of the CHS-DTRD. 


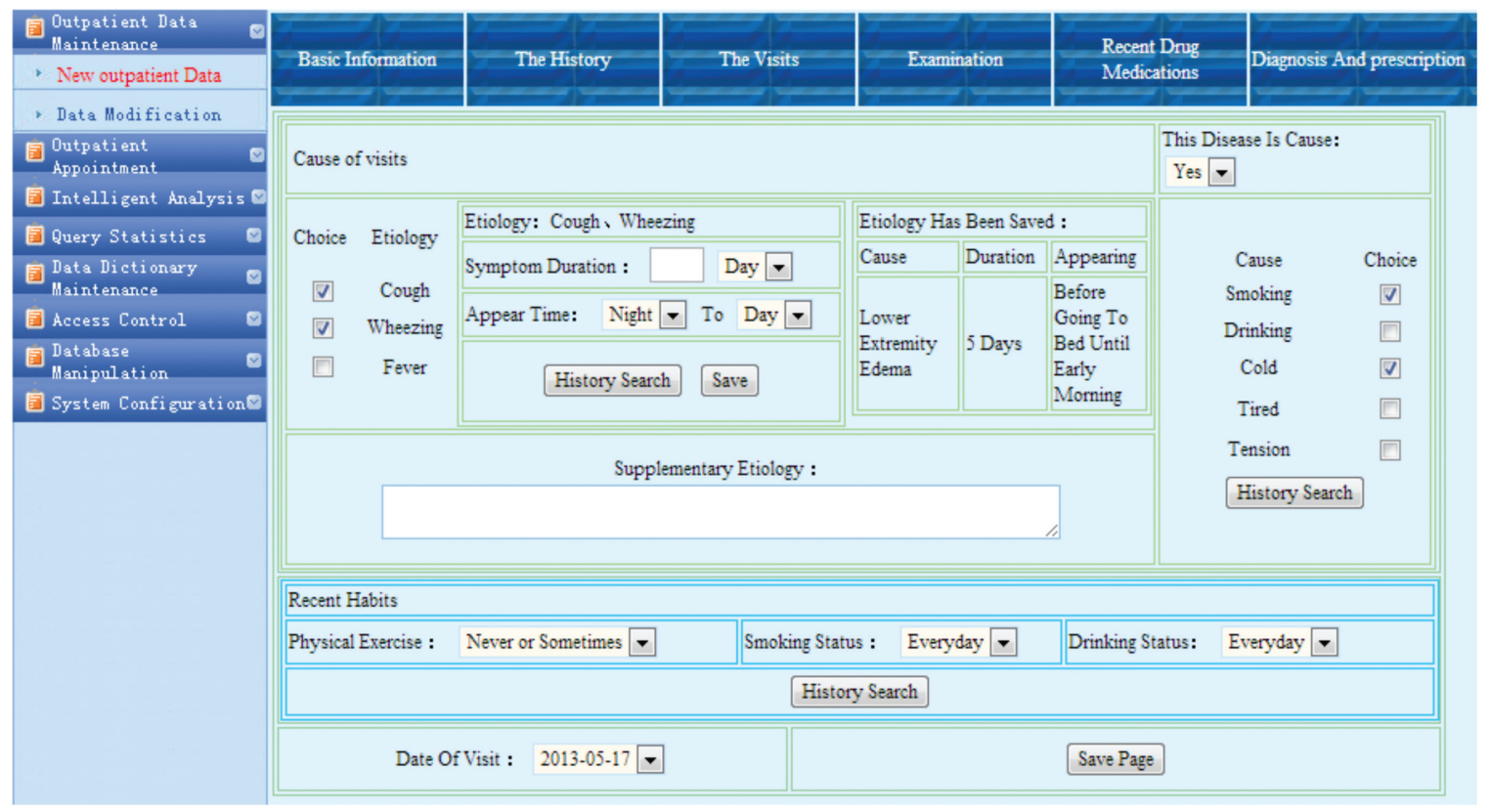

Fig. 4. Patient information input interface.

patients may make outpatient appointments by entering into the appointment interface of the system through the Internet. The system adopts a light workflow engine to distribute, dispatch, manage, and monitor the implementation of tasks. This appointment system divides users into the following three types: patient users; physician users; and system administrators. Patient users mainly use this system to query the physician and medical information, and review and amend appointment information. Physician users may provide the appointment schedule, and establish, amend, and cancel the medical records of patients. Administrators may only add, cancel, and manage the physician accounts, but not amend physician reception information and patient outpatient appointment information.

Intelligent analysis on disease risk factors: It is important for the prevention, diagnosis, and treatment of diseases to identify disease risk factors. Therefore, the module of "Intelligent Analysis for Disease Risk Factors" of this system adopted a data mining technique to conduct intelligent analysis for disease risk factors.

High quality basic data is the necessary condition for effective implementation of data mining. Therefore, this computer-assisted information system was designed to record full and complete outpatient information and control the consistency and completeness of data to facilitate the follow-up data mining work. A data mining technique was applied to identify which factor is closely related to a certain disease. Data mining is a technique aimed to make data analysis and reveal knowledge hidden in the data by appropriate machine learning algorithms, and identify knowledge and information hidden in the data-containing noise. Data mining is currently one of the most advanced methods for data analysis. The data mining technique has been widely used in medical treatment, health care, and other related fields [12]. We adopted the data mining technique for intelligent analysis on the risk factors of respiratory diseases in this article.

The module "Intelligent Analysis for Risk Factors" is composed of the following three parts: data preprocessing; knowledge mining; and knowledge visualization. Data preprocessing processes the data through data cleaning, sorting, and regularization without losing the information contained in the data. During the data mining period, rough sets theory was first used for attribute reduction, then used the association rule mining algorithm [13] to mine disease risk factors. Frequent item sets were judged according to the common frequency of 
symptoms and diagnosed disease, and based on the comparison of the minimum reliability and minimum support set in rules, to find out the association rules between these common symptoms and diseases. Knowledge visualization shows the obtained knowledge by visual graphical means.

Query and statistics: This system provides a powerful query and statistics function. Users may define query conditions and results. The results may be shown in a diagram or exported to Excel, which are easy to process. Query Statistics is also the pre-processing module of "Intelligent Analysis for Risk Factors."

This system made full use of the AJAX technique and adopted the data linkage box method. The "linkage dropdown box" refers to several drop-down lists which are combined for users to choose items one-by-one. In this way, the scope is gradually narrowed and the most accurate item is finally selected, so that users can find related information from one aspect of such information.

The linkage drop-down box has the following three advantages:

(a) improvement of program efficiency-the client end only obtains the required data from the server, thus greatly reducing the program response time;

(b) reduction of network flow-instead of sending all related information obtained to the client end at one time, it not only reduces the use of server resources, but also reduces network flow; and

(c) good user experience-it reduces the waiting time for page load.

Data dictionary maintenance: To improve extensibility, functional modularity, independence of data structure, and the generality of the system, a data dictionary system was adopted, which uses UML to analyze the system and establish modules [14]. For example, to reduce a physician's input workload, a relevant data dictionary of common values in specific fields was established, including medical history, drugs, foes, family medical history, disease incentives, allergens, causes of disease (symptoms), and physicians. The content of these fields in the data dictionary may be added, cancelled, and amended. For fields in which a specific data dictionary is available, the physicians may just choose suitable data instead of typewriting, thus improving the usability of software and making good extension of the system.

It is notable that the structure design of the data dictionary system is very important. It is essential to determine the type of data dictionary by analyzing the common data to be shared and interface to be provided by various functional modules.

Database manipulation: This system adopted a relational database. To ensure the database meets user requirements, the database structure is reasonable, and access of data is in an orderly manner to facilitate query and amendment, the following steps for design were adopted:

(a) Analyze the needs of the entire system and identify what information must be put into the database. On the basis of analysis of the data flow of the CHS-DTRD, obtain the duplicates of forms and reports required during the current data processing to define the fields and forms contained in the database.

(b) Determine the elements which should be recorded in the CHS-DTRD. List the data items which should be recorded in the system and give aside samples to facilitate the determination of data type.

(c) Identify forms and fields. Determine the entity and establish the entity relation diagram. Distribute and nominate the fields for each data sheet, designate sample data, data type, and estimate field capacity for each field. Fields and form names should be described in English and use specialized vocabularies in actual business flow.

(d) Define key code and relationships. Set the primary and external keys according to the relationship diagram, and set referential integrity according to the business operations.

(e) Data normalization. The data sheet was designed to meet the third normal form according to the scale of system users and the data needs; however, in some cases, data redundancy is added to improve the query efficiency of the database.

(f) Transaction processing. All key database manipulations of this system adopted a transaction processing mechanism to ensure the consistency of data and the stability of the system. 
Access control: Access control is extremely important for system safety. Traditional access control is realized by corresponding access right directly to users. With the popularity of the network, the structure of information resources that users can access becomes more complicated. The traditional method cannot meet the requirements of management of complicated information. Therefore, a Role-Based Access Control (RBAC) mechanism was generated [15] for this system. A role-based access control application model suitable for a medical information management system was developed by introducing the RBAC. Through the use of the RBAC, the enterprise's organizational structure may be directly reflected in the information system, which makes user management more intuitive and, to a large extent, makes access management more simplified. In practical application, the management method of access control is featured with simplicity, efficiency, and safety, which can properly meet the practical requirements.

System configuration: Configuration of operating parameters for the system may be completed through the functions provided by system configuration. System configuration provides configuration of default parameters. Configuration of default parameters may ensure normal and stable operation of the system on most occasions; in addition, the administrator may adjust parameters accordingly to respond the specific application occasion. For example, when there are more community personnel and possibly more online users, the maximum concurrent access parameter value of the system should be increased accordingly.

\section{Intellectual property}

We have obtained the software copyright for Outpatient Registration System for Respiratory Diseases V1.0 for some achievements of this study Date of Authorization: May 9, 2012; Authorization No.: 2012SR0306750.

\section{Discussion}

This article introduced the design and realization of the CHS-DTRD on the basis of AJAX and JSP. The system features low cost, convenient deployment, high stability, and strong extensibility. Thus far, the system has been adopted in clinical practice and scientific studies by the Department of Respiratory Medicine, the First Affiliated Hospital with Nanjing Medical University, and the Nanjing University of Science and Technology Hospital, and will be promoted and used in other community hospitals. The system development is significant in the following three aspects:

First, the system application is of strong pertinence, which has established the community health service-oriented outpatient application system with standardized and advanced technological diagnosis and treatment of diseases, and taken into consideration the characteristics of outpatient respiratory diseases and the particularities of community practice.

Second, this system adopts advanced technology to achieve high stability and strong extendibility. Interface matching of HIS is reserved to connect with the existing outpatient software or computer-assisted examination report system in hospitals. This makes the data collection more convenient, and simplifies the tedious input work of computer-assisted tests and examinations. The system can also realize picture storage, release, and sharing. The system interface style and operation method are consistent with common office software, and great help is provided for users, so the users can become familiar with operation of this system quickly. The query module has flexible customized retrieval function and patients registered in the database could be rapidly retrieved by their names, ID, or outpatient card numbers. Data can be exported as needed to facilitate clinical study and health statistics.

Third, analysis of disease risk factors based on the data mining technique is an important function, which plays a significant role in the prevention, diagnosis, and subsequent treatment of respiratory diseases. The decision rules for disease risk factors obtained by mining technique are scientific, objective, and practical. Through qualitative analysis of the decision rules obtained, we could determine the knowledge contained in the decision rules is consistent with human cognition.

Application of this system can make the diagnosis and treatment of respiratory diseases simplified, programmable, and intuitive in the community, and reduce physician workload, improve work efficiency, and patient satisfaction at the same time. 


\section{Conflict of interest}

The authors declare no conflict of interest.

\section{References}

1. Murray CJ, Lopez AD. Evidence-based health policy-lessons from the Global Burden of Disease Study. Science 1996;274: 740-3.

2. Cui DJ. New progress in the treatment of bronchial asthma. China J Tuberc Respir Dis 2004:27;474-6.

3. Bao Y. A practical guide to community health service. Shanghai: Shanghai Science and Technology Press, 2004.

4. Hu HP, Lei XY, Chen Q, Xie LQ. Construction of automated community health service supervision and management system. China Gen Pract 2011;14:2847-9.

5. de Leeuw R. Implementation of an electronic hospital information system: first results of a longitudinal study. Int J Int Car 2012;12.

6. Cao Y, Yang LN, Cao H. Design and development of a hospital information system in China. J Invest Med 2013;61:S10.

7. Wu YF, Sun PL, Yu HY. Design and implementation of respiratory assisted treatment system. China Dig Med 2011;6:60-2.
8. Yu H, Shi HY, Yang J, Qiu MH. Design and implementation of proxy laboratory and examination system in HIS. China Dig Med 2013;7:48-50.

9. Wu JY, Ping LD. Performance analysis on magistral application technology in Web 2.0-AJAX. Comp Eng Des 2008;29:913-4.

10. Li AQ. Application of JSP/Servlet technique in management information system. J Qinghai University (Natural Science) 2004;22:66-8.

11. Pang YJ, Fang DW. The application and development of SQL Server example, Beijing: People's Posts and Telecommunications Press, 2009.

12. Koh HC, Tan G. Data mining applications in healthcare. J Health Inf Manag 2005;19:64-72.

13. Yang DH, Kang JH, Park YB, Park YJ, Oh HS, Kim SB. Association rule mining and network analysis in oriental medicine. PLoS One 2013;8:e59241.

14. Tan M, Liu LC, Ni WC. Design and implementation of a data dictionary in information model based on UML. Comp Eng Des 2008;29:849-54.

15. Kuhn DR, Coyne EJ, Weilsities TR. Adding attributes to rolebased access control. Computer 2010;43:79-81. 